\title{
A Right Paratracheal Air Cyst Caused by Tracheal Diverticula
}

\author{
Tomohide Sato ${ }^{1}$, Yuko Sasaki ${ }^{1}$, Motohisa Yamasaki ${ }^{1}$, Mikayo Aragaki ${ }^{1}$, Sunao Mae ${ }^{1}$, \\ Tetsuya Irie $^{1}$ and Yuki Sumi ${ }^{1,2}$
}

\begin{abstract}
A 65-year-old woman presented with pain in her throat and neck. Thin-section computed tomography (CT) revealed an air-filled lesion at the right paratracheal region and two narrow connections to the trachea. Flexible bronchoscopy showed four diverticula $4-5 \mathrm{~cm}$ below the vocal cords in the right lateral part of the trachea. Consistent with the CT findings, two of the diverticula were deep. A radiological barium swallow study and an esopagogastroduodenal endoscopic examination revealed no abnormalities. We therefore believe that her right paratracheal air cyst is an extension of a tracheal diverticulum. Right-sided paratracheal air cysts at the level of the thoracic inlet are a common finding on CT and should not be confused with pneumomediastinum in order to avoid unnecessary examinations or treatments.
\end{abstract}

Key words: tracheal diverticulum, paratracheal air cyst

(Inter Med 49: 315-319, 2010)

(DOI: 10.2169/internalmedicine.49.2731)

\section{Introduction}

Paratracheal air cysts mimic pneumomediastinum, which may be caused by digestive tract perforation and may result in mediastinitis. Although right paratracheal air cysts have so far only been rarely described and very few reports have been published (2-7), paratracheal air cysts may be a common CT finding that occur in a predictable location $(1,8)$. Patients generally have a good prognosis after the diagnosis of tracheal diverticula and clinicians should become familiar with this disease entity. We herein report a patient with a right paratracheal air cyst caused by a tracheal diverticulum which was confused with pneumomediastinum.

\section{Case Report}

A 65-year-old woman was admitted to our hospital with suspected mediastinal emphysema caused by esophageal perforation due to a fish bone. She visited our hospital because she had had the pain in the throat and neck after eating alfonsin seven days prior to her admission. She had been diagnosed with paroxymal second-degree atrioventricular block Mobitz type I (Wenckebach block) ten years previously. She had also experienced discopathy three years earlier.

On physical examination she presented with painful left submandibular adenopathy. An otological evaluation revealed inflammation of the submandibular gland and salivary stone but there was no throat disorder.

Her temperature was $36.4^{\circ} \mathrm{C}$, blood pressure $114 / 72$ $\mathrm{mmHg}$, and pulse 80 beats per minute. Her lungs were clear on auscultation. Laboratory findings indicated inflammation, as her white blood cell count was 16,630 per cubic millimeter and C-reactive protein was $9.7 \mathrm{mg} / \mathrm{dL}$ (Table 1). Electrocardiography revealed an incomplete right bundle branch block. Chest radiography was normal (Fig. 1A) but computed tomography (CT) scanning of the chest revealed an air-filled lesion at the right paratracheal region (Fig. 1C), pleural thickness in the bilateral apical portions of the lung (Fig. 1B and C), and slight bronchioectasis in the right middle lobe suggesting old inflammation scars (Fig. 1D).

As we could not exclude the possibility that she had infectious mediastinitis due to esophageal perforation, she

${ }^{1}$ Internal Medicine, Nakano General Hospital, Tokyo and ${ }^{2}$ Department of Integrated Pulmonology, Graduate School of Medical and Dental Science, Tokyo Medical and Dental University, Tokyo

Received for publication July 30, 2009; Accepted for publication October 19, 2009

Correspondence to Dr. Yuki Sumi, sumi-alg@umin.ac.jp 
Table 1. Laboratory Tests on Admission

\begin{tabular}{|c|c|c|c|c|c|}
\hline \multicolumn{5}{|c|}{ Reference } & \multirow{2}{*}{ Reference } \\
\hline & on admission & range & & on admission & \\
\hline White cells (per mm³) & 16630 & $(3400-8800)$ & Creatinine $(\mathrm{mg} / \mathrm{dL})$ & 0.52 & $(0.6-1.3)$ \\
\hline Differencial count $(\%)$ & & & Sodium $(\mathrm{mmol} / \mathrm{L})$ & 138 & $(134-146)$ \\
\hline Neutrophils & 86 & $(16-60)$ & Potassium $(\mathrm{mmol} / \mathrm{L})$ & 4.4 & $(3.4-4.9)$ \\
\hline Lymphocytes & 11 & $(18-50)$ & Chloride $(\mathrm{mmol} / \mathrm{L})$ & 102 & $(98-108)$ \\
\hline Monocytes & 1 & $(1-10)$ & Calcium (mg/dL) & 9.4 & $(8.6-10.8)$ \\
\hline Eosinophils & 2 & $(1-7)$ & Lactate dehydrogenase (U/L) & 207 & $(106-220)$ \\
\hline Basophils & 0 & $(0-2)$ & Alanine aminotransferase (U/L) & 34 & $(8-40)$ \\
\hline Atypical lymphocytes & 0 & & Aspartate aminotransferase (U/L) & 47 & $(5-35)$ \\
\hline Hemoglobin $(\mathrm{g} / \mathrm{dL})$ & 11.6 & $(13.2-17.2)$ & Alkaline phosphatase & 660 & $(100-340)$ \\
\hline Hematocrit (\%) & 35.3 & $(39.2-49.2)$ & Bilirubin Total (mg/dL) & 0.84 & $(0.3-1.10)$ \\
\hline Platelet count $\left(\times 10^{4}\right.$ per $\left.\mathrm{mm}^{3}\right)$ & 25.6 & $(11.8-36.4)$ & Glucose (mg/dL) & 135 & $(60-110)$ \\
\hline Total protein $(\mathrm{g} / \mathrm{dL})$ & 8.0 & $(6.8-8.2)$ & Total Cholesterol (mg/dL) & 186 & $(130-220)$ \\
\hline Albumin (g/dL) & 3.6 & $(3.7-5.2)$ & Amylase (U/L) & 79 & $(35-120)$ \\
\hline Urea nitrogen (mg/dL) & 12 & $(5-22)$ & C-reactive protein $(\mathrm{mg} / \mathrm{dL})$ & 9.7 & $(<=0.5)$ \\
\hline
\end{tabular}

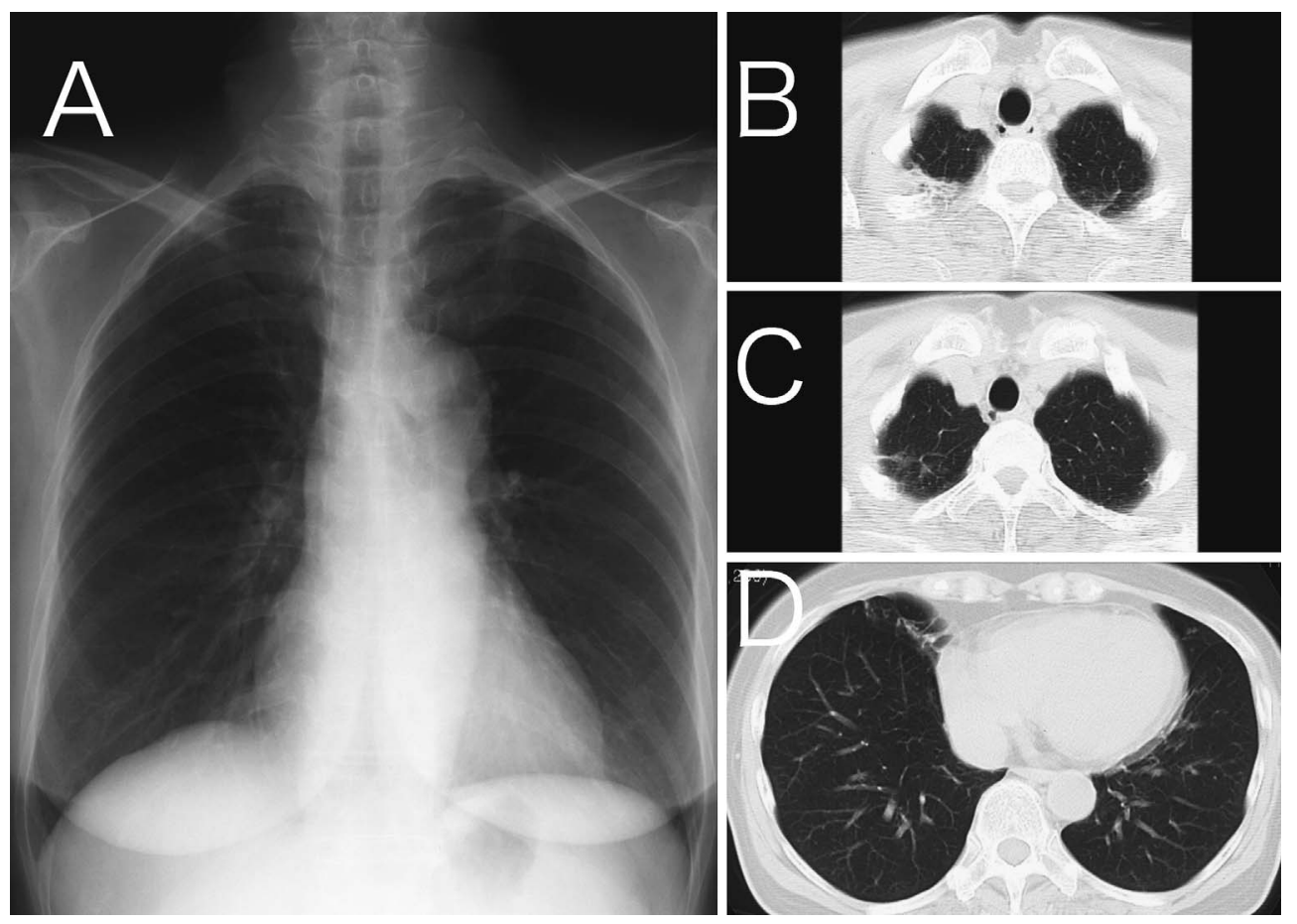

Figure 1. Chest radiography was normal (panel A). CT scanning of the chest revealed an airfilled lesion at the right paratracheal region (panel $\mathrm{C}$ ), pleural thickness in the bilateral apical portions of the lung (panels $\mathrm{B}$ and $\mathrm{C}$ ), and slight bronchioectasis in the right middle lobe, thus suggesting old inflammation scars (panel D).

fasted for a day and received fluid intravenously. She received $0.5 \mathrm{~g}$ cefazoline sodium every 12 hours for five days and $0.5 \mathrm{~g}$ cefotiam dihydrochloride every 12 hours for the subsequent twelve days. Her symptoms thereafter improved rapidly. As the Gastrografin ${ }^{\circledR}$ (Diatrizoate Meglumine and Diatrizoate Sodium Solution) swallow study performed on the fourteenth day revealed no abnormalities (Fig. 2A), antibiotics were thus discontinued and she was permitted to eat and was discharged. However, her serum C-reactive protein level did not return to the normal range as an outpatient and it took 40 days after being admitted to a hospital to reach a normal range. An esophagogastroduodenal endoscopic examination performed on an outpatient basis revealed no ab- normalities (Fig. 2B); however, a follow-up thin-section CT still revealed an air-filled lesion at the right paratracheal region in addition to two narrow connections to the trachea (Fig. 3A and B). Flexible bronchoscopy showed four tracheal diverticula. Consistent with CT findings, two deep diverticula $4-5 \mathrm{~cm}$ below the vocal cords were observed (Fig. 3C, D, and E). A shaded-surface-display threedimensional image of trachea clearly revealed the anatomic relationship between the trachea and the air cyst (Fig. 4). Currently, she routinely visits the clinic without symptoms and her chest CT always shows an unchanged right paratracheal air cyst. 


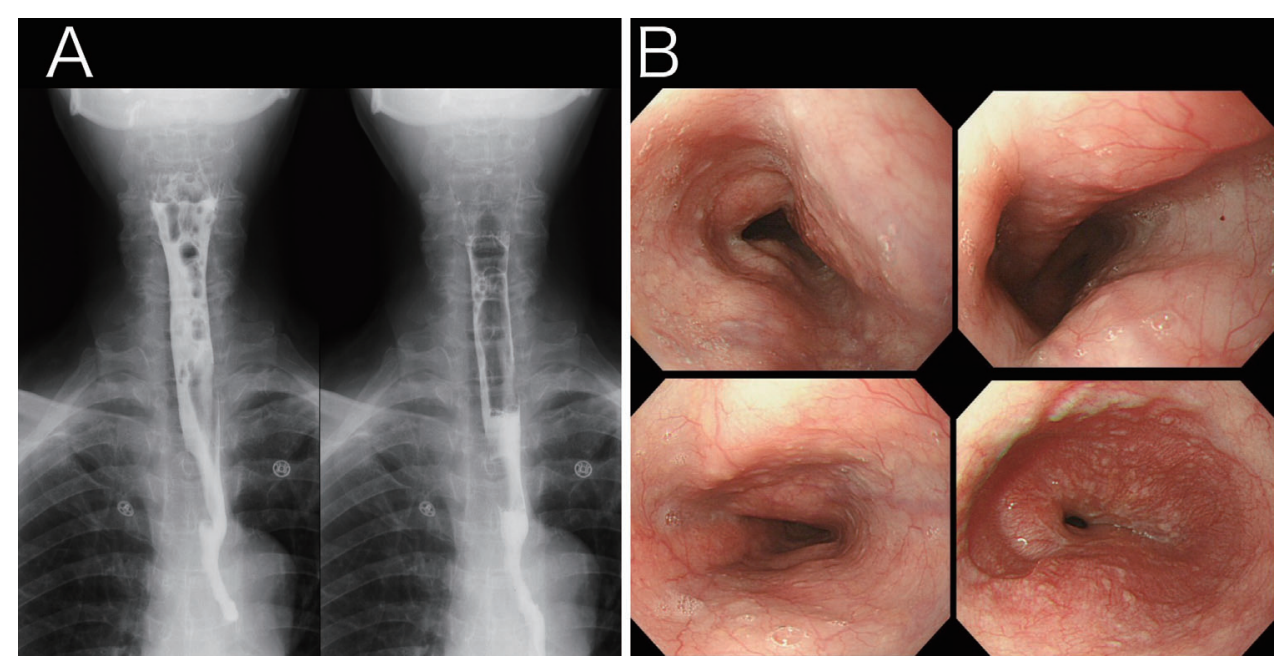

Figure 2. A Gastrografin ${ }^{\circledR}$ (Diatrizoate Meglumine and Diatrizoate Sodium Solution) swallow study (panel A) and esophagogastroduodenal endoscopic examination (panel B) revealed no abnormalities.

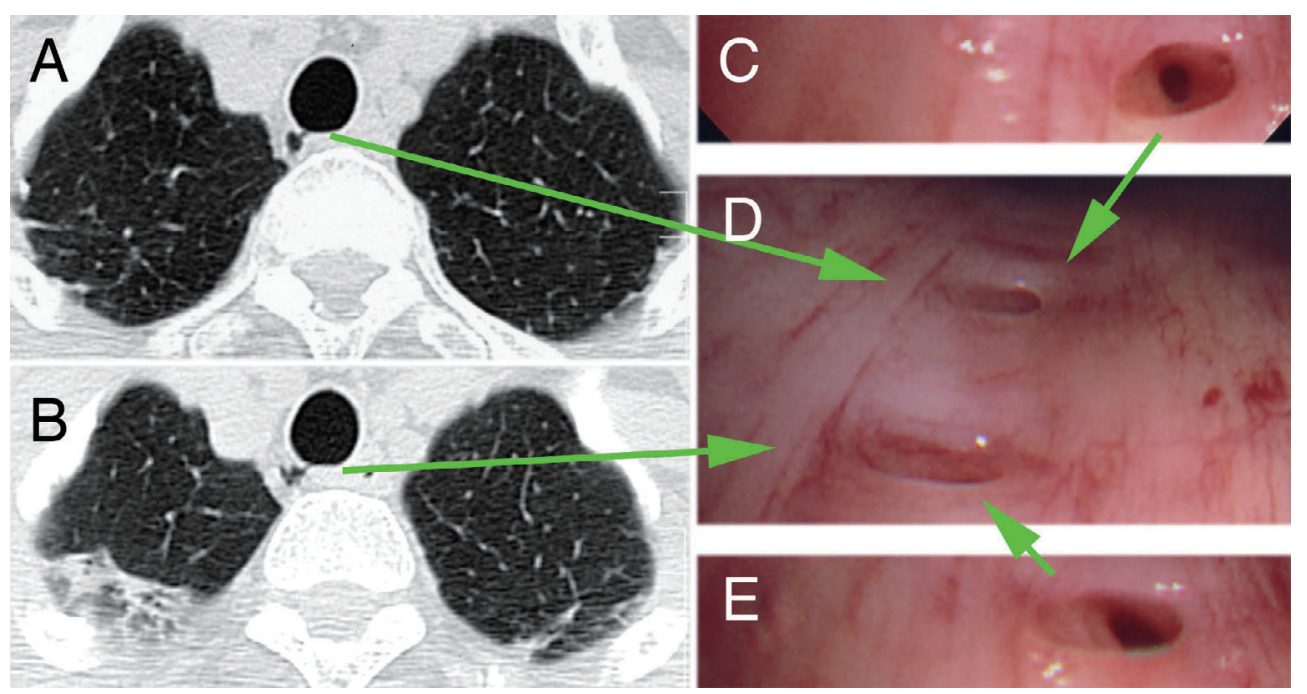

Figure 3. Thin-section CT revealed an air-filled lesion at the right paratracheal region. (panel A and B). Two narrow connections to trachea were also visible. Flexible bronchoscopy showed four tracheal diverticula. Consistent with the CT findings, two diverticula were deep (panel C, D and E).

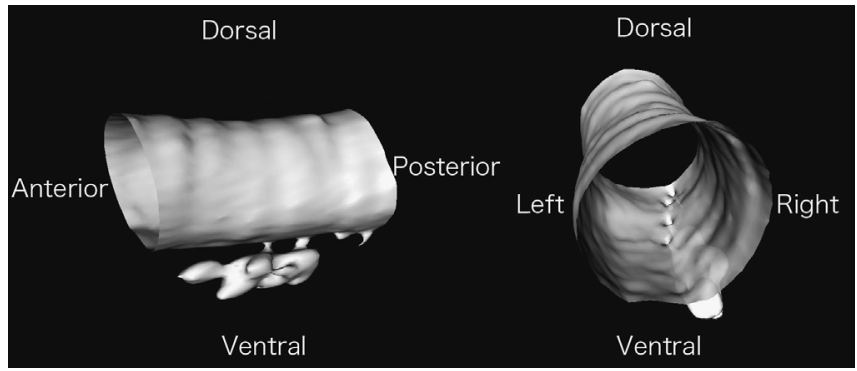

Figure 4. Shaded-surface-display three-dimensional image of the trachea clearly revealed the anatomic relationship between the trachea and the air cyst.

Discussion

There are several published case reports (2-7) concerning right paratracheal air cysts. In these reports, the cysts were lined with ciliated columnar epithelia and they communicated with the trachea; therefore, they could be considered as tracheal diverticula. Other causes of paratracheal air collection include laryngocele, pharyngocele, Zenker's diverticulum, apical hernia of the lung, and apical paraseptal blebs or bullae. Laryngocele, pharyngoceles and Zenker's diverticula are usually identified by a barium examination. An apical hernia has continuity with the body of the lung and apical paraseptal blebs or bullae are air cysts within the lung. These conditions can be easily recognized on CT scans. 
A previous study reported that paratracheal air cysts were visible in only $14 \%$ of the study subjects on chest radiographs (8). On CT images, $98.5 \%$ of the paratracheal air cysts were located in the right posterolateral region and $1.5 \%$ were in the left paratracheal region. The cyst was at the level of the T1 vertebral body in $29 \%$ of the patients, at the Tl-T2 level in 17\%, at the T2 level in $42 \%$, and at the T2-T3 level in $12 \%$. On thick-section CT, communication between the air cyst and trachea was observed in only $8 \%$ of the patients with paratracheal air cysts.

A tracheal diverticulum is a benign entity characterized by single or multiple invaginations of the tracheal wall. Tracheal diverticula resemble laryngeal diverticula, which are a more common disorder and may share the same pathogenic mechanism. The overall prevalence of tracheal diverticula is about $1 \%$ according to an autopsy series by MacKinnon (9), who identified eight cases in 867 routine serial autopsies. In the present case, the diverticulum occurred on the right side, with the tracheal mucosa protruding through an area of weakness laterally between the cartilaginous rings or posterolaterally through the tracheal muscle. In the lumen of the trachea at the junction of the cartilage rings and tracheal muscle, a pinhead-sized opening was observed opposite the midpoint of each cyst.

Bronchoscopy is useful in confirming the diagnosis, although cases exist in which the communication was not visible, e.g., diverticula with a very narrow opening (6).

Two types of tracheal diverticula exist -- congenital and acquired. These types vary in regard to location, characteristics, and histology. A congenital tracheal diverticulum is small and is generally located approximately $4-5 \mathrm{~cm}$ below the vocal cords or a few centimeters above the carina in the right lateral part of the trachea (10-12). This type is more common in mans than in womans, and it is considered to be a supernumerary, malformed branch of the trachea.

Diverticula possess a complete tracheal anatomy (respiratory epithelium, smooth muscle, and cartilage) and are usually filled with mucus. Acquired tracheal diverticula (10-12) may appear at any level, although they are more common in the posterolateral region between the extrathoracic and intrathoracic trachea at the entrance to the thorax, as a result of the anatomical features of this area. Acquired tracheal diverticula can develop due to an increase in the tracheal intraluminal pressure caused by a chronic cough or by obstructive lung disease with emphysema combined with a weakened musculature of the trachea wall due to repeated respiratory infections. Acquired tracheal diverticula are larger than the congenital type and the lesion wall consists of only respiratory epithelium without any cartilaginous or smooth muscular elements. Although the classification of tracheal diverticula as either congenital or acquired is widely used, others have classified air cysts of tracheal origin into tracheogenic cysts (with muscle and cartilage in the wall and of embryonic origin), tracheoceles (one single formation larger than 2 $\mathrm{cm}$ ), and diverticula (multiple formations smaller than 2 $\mathrm{cm})$. Tracheoceles and diverticula lack a muscular frame (8).
The association of marked dilatation of the trachea and main bronchi combined with tracheal diverticula, bronchiectasis, and recurrent lower respiratory tract infections is termed Mounier-Kuhn syndrome.

Tracheal diverticula are usually asymptomatic and tend to be incidentally detected. Nevertheless, a tracheal diverticulum can act as a cavity full of secretions and clinically manifest as a chronic cough, repeated episodes of respiratory infections, hemoptysis, stridor, or dyspnea. Rarely, a large diverticulum can compress the recurrent laryngeal nerve and result in dysphonia (10). There has been a case report of respiratory distress due to selective intubation of the diverticulum and perforation of the lesion during airway manipulation resulting in a pneumomediastinum (13).

Surgical treatment of a tracheal diverticulum is rarely advocated. Conservative measures such as antibiotics, mycolytic agents and physiotherapy are generally proposed, especially in older patients. Surgery should be reserved for young or symptomatic patients. Surgical options include laser cauterization via rigid bronchoscopy or open repair via a lateral cervical approach.

In the present case the patient's chief complaint was throat and neck pain after eating fish, which initially seemed to indicate a perforated esophagus by a fish bone; however, we believe the pain was caused by inflammation of the submandibular gland. In this case the paratracheal air cyst was incidentally detected on a chest CT examination and was not evident on a chest radiograph. Flexible bronchoscopy and chest CT showed four tracheal diverticula located 4-5 $\mathrm{cm}$ from the vocal cords on the right lateral wall of the trachea, two of which communicated to the trachea as a paratracheal air cyst. As our case had multiple diverticula with a characteristic distribution, we believe that the paratracheal air cyst in this case was therefore really congenital tracheal diverticula, although we do not have any pathological evidence for this. She did not have any medical history of either chronic cough or lung disease to increase the intraluminal pressure or any repeated respiratory infections which could weaken the musculature of the trachea wall, thus leading to the development of acquired tracheal diverticula.

Although her painful left submandibular adenopathy rapidly improved, the inflammation represented by her serum C-reactive protein level persisted for 40 days. She therefore may have experienced a mediastinal infection through the tracheal diverticula.

Right paratracheal air cysts had been thought to be rarely encountered, but recent radiologic studies showed their prevalence to be $2 \%$ (8) or $3.7 \%$ (1) in patients undergoing CT. Due to the increasing availability and technical progress of CT, the frequency of detecting paratracheal air cysts is therefore expected to increase. In the present case the right paratracheal air space was very difficult to estimate in detail based on only conventional CT without thin slice CT. Paratracheal air cysts can mimic pneumomediastinum. However, such paratracheal air cysts are mostly located at the thoracic inlet level and at the right posterolateral aspect of the tra- 
chea and are therefore usually recognizable. Threedimensional reconstruction CT may therefore be a useful diagnositic modality for determining the extent of the diverticulm. Once diagnosed, the majority of such patients require no specific intervention. Clinicians should therefore include tracheal diverticula in the differential diagnosis of pa- tients presenting with either respiratory symptoms or right paratracheal air cysts.

\section{Acknowledgement}

The authors would like to thank Mr. Hiroyasu Ina for his valuable assistance with the radiology procedures.

\section{References}

1. Buterbaugh JE, Erly WK. Paratracheal air cysts: a common finding on routine CT examinations of the cervical spine and neck that may mimic pneumomediastinum in patients with traumatic injuries. AJNR Am J Neuroradiol 29: 1218-1221, 2008.

2. Tanaka H, Igarashi T, Teramoto S, Yoshida Y, Abe S. Lymphoepithelial cysts in the mediastinum with an opening to the trachea. Respiration 62: 110-113, 1995.

3. Tanaka H, Mori Y, Kurokawa K, Abe S. Paratracheal air cysts communicating with the trachea: CT findings. J Thorac Imaging 12: 38-40, 1997.

4. Infante M, Mauavelli F, Valente M, Alloisio M, Preda F, Ravasi G. Tracheal diverticulum: a rare cause and consequence of chronic cough. Eur J Surg 160: 315-316, 1994.

5. Pinot D, Breen D, Peloni JM, Gaubert JY, Dutau H, Vervloet D. An incidental finding in a 34-year-old male under investigation for haemoptysis. Diagnosis: The radiological and endoscopic images demonstrate a complex defect along the posterior tracheal wall consistent with acquired tracheal diverticulum. Eur Respir J 33: 1227-1229, 2009.

6. Kokkonouzis I, Haramis D, Kornezos I, Moschouris H, Katsenos $\mathrm{S}$, Bouchara S. Tracheal diverticulum in an asymptomatic male: a case report. Cases J 1: 181, 2008.

7. Soto-Hurtado EJ, Peñuela-Ruíz L, Rivera-Sánchez I, TorresJiménez J. Tracheal diverticulum: a review of the literature. Lung 184: 303-307, 2006.

8. Goo JM, Im JG, Ahn JM, et al. Right paratracheal air cysts in the thoracic inlet: clinical and radiologic significance. Am J Roentgenol 173: 65-70, 1999.

9. MacKinnon D. Tracheal diverticula. J Pathol Bacteriol 65: 513517, 1953.

10. Caversaccio MD, Becker M, Zbären P. Tracheal diverticulum presenting with recurrent laryngeal nerve paralysis. Ann Otol Rhinol Laryngol 107: 362-364, 1998.

11. Hernández JM, Pérez L, Batista JJ, Gonzalvo F. Intratracheal diverticulum. J Bronchol 12: 104-105, 2005.

12. Restrepo S, Villamil MA, Rojas IC, et al. Association of two respiratory congenital anomalies: tracheal diverticulum and cystic adenomatoid malformation of the lung. Pediatr Radiol 34: 263266, 2004.

13. Möller GM, ten Berge EJ, Stassen CM. Tracheocele: a rare cause of difficult endotracheal intubation and subsequent pneumomediastinum. Eur Respir J 7: 1376-1377, 1994.

(C) 2010 The Japanese Society of Internal Medicine http://www.naika.or.jp/imindex.html 